\title{
Static and dynamic properties of stretched water
}

\author{
Paulo A. Netz ${ }^{\text {a) }}$ \\ Departamento de Química, Universidade Luterana do Brasil, 92420-280, Canoas, RS, Brazil
}

Francis W. Starr

Polymers Division and Center for Theoretical and Computational Materials Science, National Institute

of Standards and Technology, Gaithersburg, Maryland 20899

$\mathrm{H}$. Eugene Stanley

Center for Polymer Studies and Department of Physics, Boston University, Boston, Massachusetts 02215

\author{
Marcia C. Barbosa \\ Center for Polymer Studies and Department of Physics, Boston University, Boston, Massachusetts 02215 \\ and Instituto de Física, Universidade Federal do Rio Grande do Sul, Caixa Postal 15051, \\ 91501-970, Porto Alegre, RS, Brazil
}

(Received 12 February 2001; accepted 9 April 2001)

\begin{abstract}
We present the results of molecular dynamics simulations of the extended simple point charge model of water to investigate the thermodynamic and dynamic properties of stretched and supercooled water. We locate the liquid-gas spinodal, and confirm that the spinodal pressure increases monotonically with $T$, supporting thermodynamic scenarios for the phase behavior of supercooled water involving a "non-reentrant" spinodal. The dynamics at negative pressure show a minimum in the diffusion constant $D$ when the density is decreased at constant temperature, complementary to the known maximum of $D$ at higher pressures. We locate the loci of minima of $D$ relative to the spinodal, showing that the locus is inside the thermodynamically metastable regions of the phase diagram. These dynamical results reflect the initial enhancement and subsequent breakdown of the tetrahedral structure and of the hydrogen bond network as the density decreases. (C) 2001 American Institute of Physics. [DOI: 10.1063/1.1376424]
\end{abstract}

\section{INTRODUCTION}

Water is an important liquid in nature, and is also fundamental in chemical and technological applications. Although the individual water molecule has a simple chemical structure, water is considered a complex fluid because of its anomalous behavior. ${ }^{1-5}$ It expands on freezing and, at a pressure of $1 \mathrm{~atm}$, the density has a maximum at $4{ }^{\circ} \mathrm{C}$. Additionally, there is a minimum of the isothermal compressibility at $46^{\circ} \mathrm{C}$ and a minimum of the isobaric heat capacity at $35^{\circ} \mathrm{C} .{ }^{6}$ These anomalies are linked with the microscopic structure of liquid water, which can be regarded as a transient gel-a highly associated liquid with strongly directional hydrogen bonds. ${ }^{7,8}$ Each water molecule acts as both a donor and an acceptor of bonds, generating a structure that is locally ordered, similar to that of ice, but maintaining the long-range disorder typical of liquids. Despite the extensive work that has been done on water, many aspects of its behavior remain unexplained.

Several scenarios have been proposed to account for the anomalous behavior of the thermodynamic response functions on cooling, each predicting a different behavior for the liquid spinodal, the line of the limit of stability separating the region where liquid water is metastable from the region where the liquid is unstable. (i) According to the stabilitylimit conjecture, ${ }^{9,10}$ the pressure of the spinodal line should decrease on cooling, become negative, and increase again

${ }^{a)}$ Electronic mail: netz@if.ufrgs.br after passing through a minimum. It reenters the positive pressure region of the phase diagram at a very low temperature, thereby giving rise to a line of singularities in the positive pressure region, and consequently the increase in the thermodynamic response functions on cooling in the anomalous region is due to the proximity of this reentrant spinodal. (ii) The critical point hypothesis, ${ }^{11-16}$ proposes a new critical point at the terminus of a first-order phase transition line separating two liquid phases of different density. The anomalous increases of the response functions, compressibility, specific heat, and volume expansivity, is interpreted in terms of this critical point. (iii) The singularity-free hypothesis ${ }^{8,17,18}$ proposes that actually there is no divergence close to the anomalous region; the response functions grow on lowering temperature but remain finite, attaining maximum values. Both hypotheses are consistent with a nonreentrant spinodal, and so unambiguously identifying the spinodal can rule out at least one scenario.

Water properties and anomalies can be strongly influenced by the physical or chemical properties of the medium. ${ }^{1-4,19,20}$ The effect not only of applied pressure, but also of negative pressure ("stretching"), is remarkable. The study of the behavior of this fluid under negative pressures is relevant not only from the academic point of view, but also for realistic systems. For example, negative pressures are observed, ${ }^{21}$ and seem to play an important role in the mechanism of water transport in plants. Therefore, properties that modify the structure of water, especially if this modification 
is similar to the effect of stretching (as is the case in some hydrogels ${ }^{19}$ ), also influence its dynamical behavior.

Dynamic properties, such as the diffusion constant, have been studied in detail for water systems at atmospheric and at high positive pressures, both experimentally ${ }^{22,23}$ as well as by computer simulations. ${ }^{24-30}$ The increase of pressure increases the presence of defects and of interstitial water molecules in the network. ${ }^{25}$ They disrupt the tetrahedral local structure, weakening the hydrogen bonds, and thus increasing the diffusion constant. ${ }^{29,30}$ However, a further increase in the pressure leads to steric effects which work in the direction of lowering the mobility. The interplay of these factors leads to a maximum in the diffusion constant ${ }^{29,30}$ at some high density $\rho_{\max }$. Above this density (or corresponding pressure), the diffusion of water is in some sense like that of a normal liquid, controlled by hindrance, with the hydrogen bonds playing a secondary role. However, the behavior at very low $\rho$ is less well understood.

While there are a large number of intermolecular potential functions used to simulate water, each of which gives slightly different results, the overall thermodynamic picture obtained from these models is generally very similar. Since dynamic properties are particularly sensitive to the potential choice, we use the extended simple point charge (SPC/E) potential, because it reproduces both the maximum in diffusivity under pressure as well as the power-law behavior of dynamic properties on cooling. Hence we expect our results will be valuable for understanding the properties of water at negative pressure, where simulations are particularly important since experiments are very difficult to perform in this region.

In this paper, we study how the thermodynamics and the dynamics of low-temperature water are affected by the decrease of the density. We perform molecular dynamics simulations of the SPC/E model of water in the range $210 \mathrm{~K}$ $<T<280 \mathrm{~K}$ and $0.825 \mathrm{~g} / \mathrm{cm}^{3}<\rho<0.95 \mathrm{~g} / \mathrm{cm}^{3}$. State points in this range have negative pressure, and are either a metastable stretched liquid, or a phase separated liquid-gas mixture. Additional details of the simulation procedure can be found in Ref. 29. Table I shows the thermodynamic and dynamic properties of the simulated state points as well as the time needed for equilibration and production runs. At the lowest $T$ studied, the production runs are not long enough to reliably estimate $D$.

\section{LOCATION OF THE SPINODAL}

The effect of negative pressure is still not fully understood, and only a few experimental works ${ }^{31,32}$ and simulations $^{11,15,28,29,33-35}$ have been performed on transport properties of stretched water. In this negative pressure region of the phase diagram the system is metastable, and becomes unstable beyond the spinodal line, so locating the spinodal we can ensure that our simulated state points lie in the metastable and not in the unstable region. Moreover, the shape of the spinodal can test the stability-limit conjecture against the critical point hypothesis and the singularity-free interpretation, so we first locate the density and pressure of the spinodal, which we denote $\rho_{\mathrm{sp}}(T)$ and $P_{\mathrm{sp}}(T)$, respectively.
TABLE I. Temperature, density, diffusion constant, potential energy, pressure, equilibration $t_{\mathrm{eq}}$, and production $t_{\mathrm{pr}}$ times. The uncertainty in $D$ is roughly \pm 0.008

\begin{tabular}{|c|c|c|c|c|c|c|}
\hline $\mathrm{T}(\mathrm{K})$ & $\rho\left(\mathrm{g} / \mathrm{cm}^{3}\right)$ & $U(\mathrm{~kJ} / \mathrm{mol})$ & $P(\mathrm{MPa})$ & $D\left(10^{-5} \mathrm{~cm}^{2} / \mathrm{s}\right)$ & $\begin{array}{c}t_{\mathrm{eq}} \\
(\mathrm{ns})\end{array}$ & $\begin{array}{c}t_{\mathrm{pr}} \\
(\mathrm{ns})\end{array}$ \\
\hline \multirow[t]{6}{*}{280} & 0.825 & -46.80 & $-217 \pm 18$ & 1.237 & 1.5 & 1.5 \\
\hline & 0.850 & -47.01 & $-239 \pm 19$ & 1.359 & 1.5 & 1.5 \\
\hline & 0.875 & -47.32 & $-230 \pm 17$ & 1.281 & 2 & 2 \\
\hline & 0.900 & -47.75 & $-204 \pm 24$ & 1.261 & 1.5 & 1.5 \\
\hline & 0.925 & -47.90 & $-172 \pm 29$ & 1.234 & 1.5 & 1.5 \\
\hline & 0.950 & -48.12 & $-133 \pm 17$ & 1.068 & 1.5 & 1.5 \\
\hline \multirow[t]{5}{*}{260} & 0.825 & -48.27 & $-221 \pm 20$ & 0.589 & 1.5 & 1.5 \\
\hline & 0.850 & -48.53 & $-261 \pm 17$ & 0.634 & 1.5 & 1.5 \\
\hline & 0.875 & -48.88 & $-257 \pm 19$ & 0.531 & 2 & 4 \\
\hline & 0.900 & -49.23 & $-231 \pm 20$ & 0.527 & 2 & 4 \\
\hline & 0.925 & -49.48 & $-191 \pm 21$ & 0.500 & 2 & 4 \\
\hline \multirow[t]{5}{*}{250} & 0.825 & -49.12 & $-227 \pm 11$ & 0.353 & 2 & 2 \\
\hline & 0.850 & -49.28 & $-273 \pm 15$ & 0.346 & 2 & 3 \\
\hline & 0.875 & -49.76 & $-271 \pm 18$ & 0.298 & 2 & 3 \\
\hline & 0.900 & -50.03 & $-244 \pm 17$ & 0.295 & 2 & 3 \\
\hline & 0.950 & -50.40 & $-148 \pm 18$ & 0.281 & 1 & 1.5 \\
\hline \multirow[t]{5}{*}{240} & 0.825 & -49.99 & $-230 \pm 19$ & 0.195 & 5 & 5 \\
\hline & 0.850 & -50.17 & $-263 \pm 27$ & 0.168 & 5 & 5 \\
\hline & 0.875 & -50.51 & $-282 \pm 18$ & 0.148 & 5 & 5 \\
\hline & 0.900 & -50.98 & $-258 \pm 17$ & 0.126 & 5 & 5 \\
\hline & 0.925 & -51.30 & $-213 \pm 19$ & 0.122 & 5 & 5 \\
\hline \multirow[t]{4}{*}{230} & 0.850 & -51.06 & $-278 \pm 16$ & 0.067 & 5 & 5 \\
\hline & 0.875 & -51.28 & $-300 \pm 18$ & 0.060 & 5 & 5 \\
\hline & 0.900 & -51.80 & $-272 \pm 16$ & 0.044 & 5 & 5 \\
\hline & 0.925 & -52.01 & $-212 \pm 17$ & 0.048 & 10 & 10 \\
\hline \multirow[t]{3}{*}{210} & 0.850 & -52.73 & $-314 \pm 15$ & $\cdots$ & 10 & 10 \\
\hline & 0.875 & -52.92 & $-348 \pm 21$ & $\ldots$ & 10 & 50 \\
\hline & 0.925 & -53.54 & $-211 \pm 24$ & $\ldots$ & 10 & 10 \\
\hline
\end{tabular}

We consider the $P-\rho$ behavior along isotherms. For each $T$, there is a minimum in $P$ at $\rho \approx 0.86 \mathrm{~g} / \mathrm{cm}^{3}$. At lower $\rho, P$ increases, signaling cavitation of the liquid. For densities below $\rho_{\text {sp }}, K_{T} \equiv \rho^{-1}(\partial \rho / \partial P)_{T}$ becomes negative, so the liquid is unstable with respect to the vapor phase. Therefore, the locus of infinite compressibility (minima of Fig. 1) locates the spinodal line, and sets the density below which our simulated state points are no longer thermodynamically stable.

We confirm which state points are single phase and which are phase separated by calculating the oxygen-oxygen partial structure factor,

$$
S(q)=\frac{1}{N} \sum_{j, k}^{N} e^{-i \mathbf{q} \cdot\left(\mathbf{r}_{j}-\mathbf{r}_{k}\right)} .
$$

Figure 2 shows $S(q)$ at the lowest $T(T=210 \mathrm{~K})$ for each density simulated. At the lowest density, $\rho=0.85 \mathrm{~g} / \mathrm{cm}^{3}$, $S(q)$ for $q \lesssim 10 \mathrm{~nm}^{-1}$ increases significantly, signaling the presence of large-scale structure in the system due to phase separation of liquid and gas. Similar behavior is observed for other state points that would appear to be in the unstable regime from Fig. $1 .^{36}$

To estimate the spinodal from the $P-\rho$ isotherms of Fig. 1 , we fit each isotherm with a fifth-order polynomial and calculate $P_{\mathrm{sp}}(T)$ and $\rho_{\mathrm{sp}}(T)$ by the minimum of the polyno- 


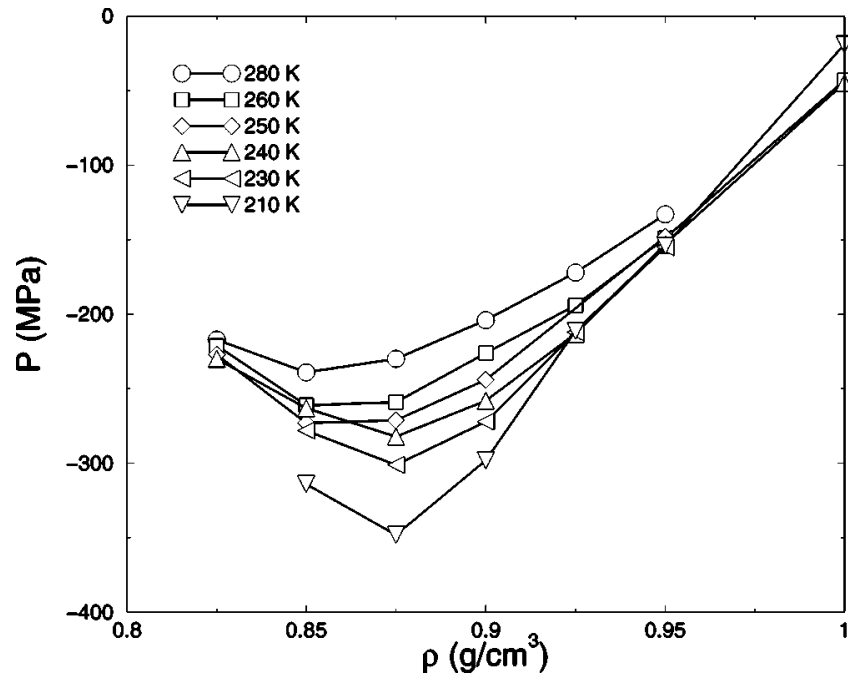

FIG. 1. Dependence of the pressure on the density, along isotherms, for the simulations reported here.

mial fit. We find that $\rho_{\mathrm{sp}}(T)$ occurs in the narrow range $0.853<\rho_{\mathrm{sp}}<0.874$, and so the spinodal density is nearly $T$ independent. Our results indicate that $P_{\mathrm{sp}}(T)$ decreases monotonically with decreasing $T$ (Fig. 3), ruling out the possibility of a reentrant spinodal in the temperature range studied.

We also compare our estimate of the spinodal with estimates from two other sources for the SPC/E potential: (i) an estimate of the upper bound on $P_{\mathrm{sp}}(T),{ }^{27}$ and (ii) the spinodal estimated by an approximate analytic equation of state (EOS) ${ }^{16}$ Figure 3 shows that our estimate of $P_{\mathrm{sp}}(T)$ is less than (and hence consistent with) the upper bound estimate of Ref. 27. The spinodal obtained from the EOS in Ref. 16 is quantitatively reasonable for $T \geqslant 280 \mathrm{~K}$, but decreases far more quickly with decreasing $T$ (reaching $\approx-2 \mathrm{GPa}$ at $T$ $=210 \mathrm{~K})$ than our simulations indicate. This dramatic decrease likely results from the fact that the fitting procedure requires $P$ along a reference isotherm, which was performed

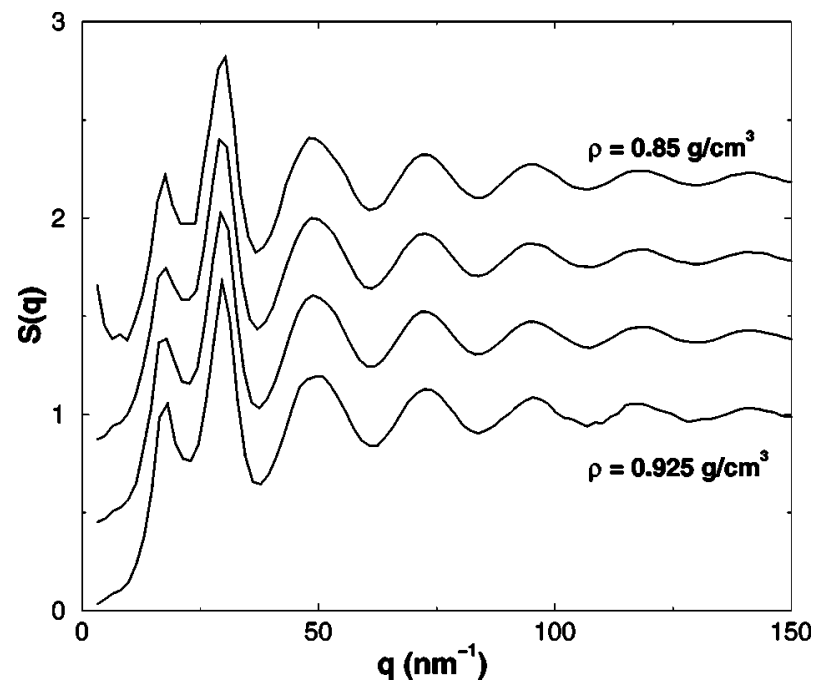

FIG. 2. At $T=210 \mathrm{~K}$, the structure factor $S(q)$. Note the increase of $S(q)$ at small $q$ at $\rho=0.85 \mathrm{~g} / \mathrm{cm}^{3}$, indicating phase separation.

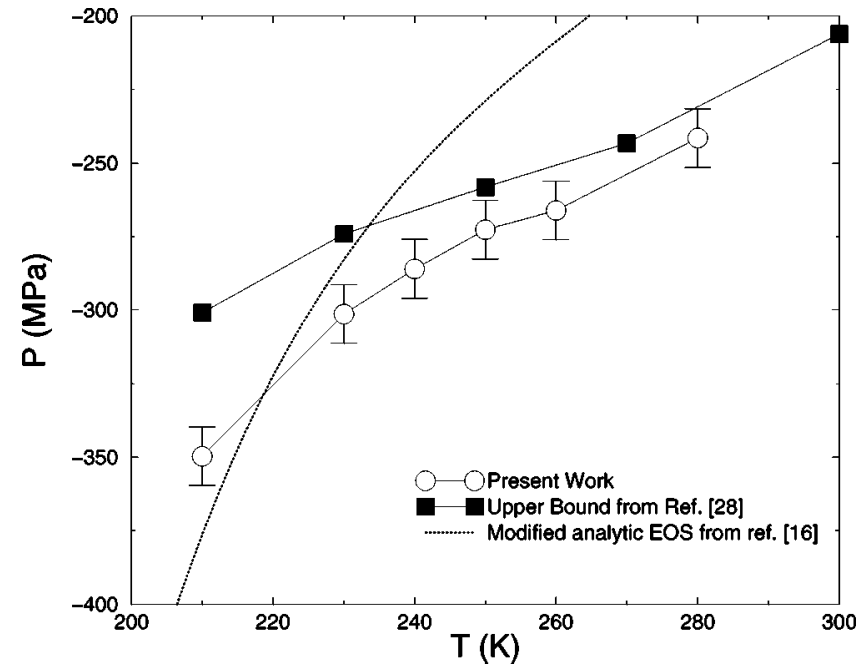

FIG. 3. Location of the spinodal $(\bigcirc)$, showing no evidence for reentrant behavior. For comparison, we also show the upper bound on the spinodal (覀) from Ref. 27 and the estimated spinodal (dotted line) from the analytic free energy expression of Ref. 16.

at $T=300 \mathrm{~K}$ in the density range $0.95<\rho<1.4 \mathrm{~g} / \mathrm{cm}^{3}$. This fit is not reliable outside of this density range, where the spinodal lies, causing an erroneous spinodal estimate. These errors are compounded as the fit is pushed further from the reference $T$. To improve the estimate, we repeat the fitting procedure using a new reference isotherm $T=210 \mathrm{~K}$, and expand the density range down to $\rho=0.85 \mathrm{~g} / \mathrm{cm}^{3}$. The results of this fit are shown in Fig. 3, and we obtain more reasonable agreement with our direct simulation results. Reference 16 also used this EOS to predict a liquid-liquid phase transition for $T \leqq 130 \mathrm{~K}$; this prediction should not be affected by using data at lower $\rho$, since the transition lies within the density range of the original fits.

\section{DYNAMIC PROPERTIES}

Having established the limit of stability at negative pressures, we next analyze the dynamics of stretched water. The effect of extreme conditions on the flow of the liquid we assess by calculating the diffusion constant $D$, defined by the asymptotic value of the slope of the mean square displacement vs time obtained by linear regression. We show $D$ along isotherms in Fig. 4. For $T \leqslant 260 \mathrm{~K}, D$ has a minimum value at $\rho \approx 0.9 \mathrm{~g} / \mathrm{cm}^{3}$, which becomes more pronounced at lower $T$ [Fig. 4(b)]. This behavior can be understood considering the structural changes that occur with decreasing density. At low $T$, the decreased density enhances the local tetrahedral ordering, which leads to a decrease in $D$. Further decreases in density reduce the stability of the tetrahedral structure and cause an increase of $D$.

The location of the minimum is near the ice Ih density $\approx 0.915 \mathrm{~g} / \mathrm{cm}^{3}$, which is the density where the perfect tetrahedral order occurs. The behavior of the minimum of $D$, $D_{\min }(T)$, complements the known behavior of $D_{\max }(T)$ for the same model, ${ }^{29,30,35}$ where a maximum occurs due to breaking hydrogen bonds at high pressure; the density of the $D_{\min }(T)$ increases slightly with increasing $T$, while the density of $D_{\max }(T)$ decreases with increasing $T{ }^{30}$ This is ex- 

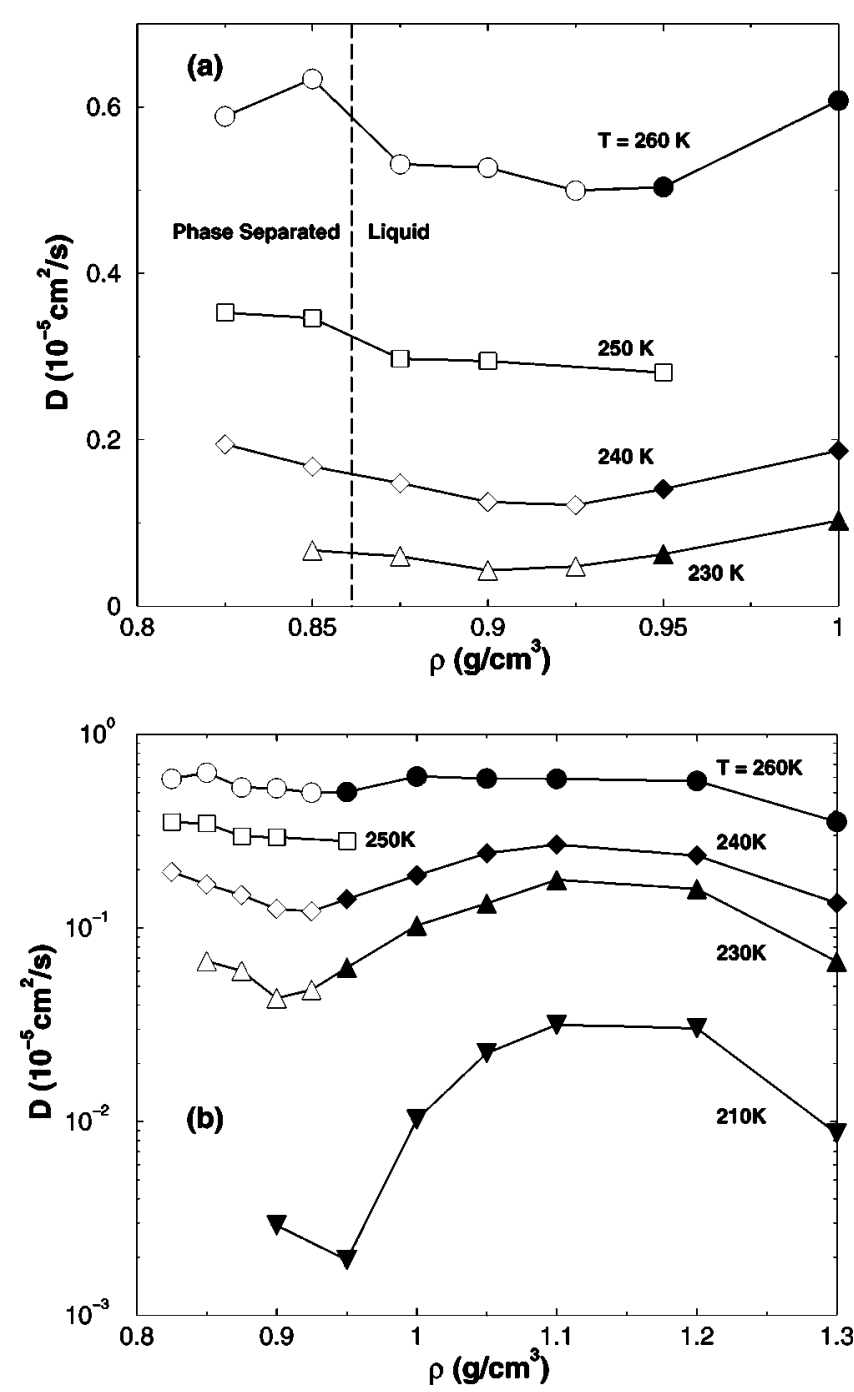

FIG. 4. (a) Dependence of the diffusion constant $D$ on $\rho$ along isotherms (for $\rho \leqslant 1.0 \mathrm{~g} / \mathrm{cm}^{3}$ ). Open symbols are the new simulations we report, and closed symbols are from Ref. 29. The dotted line separates liquid state points from phase separated state points, but is not an indication of the exact $\rho_{\text {sp }}(T)$, which varies slightly with $T$. (b) Full $\rho$ dependence of $D$, also showing the maxima.

pected, since the range of densities where anomalous behavior occurs expands with decreasing $T$. We show the loci of $D_{\min }(T), D_{\max }(T)$, along with the spinodal and the temperatures of maxima density, $T_{M D}(P)$ in Fig. 5.

\section{CONCLUSIONS}

Water exhibits a very complex structure and its properties and anomalies are strongly influenced by variations of pressure. For high densities $\left(\rho>\rho_{\max }\right)$, water behaves as a normal liquid and the decrease of $D$ with increasing pressure is governed by steric effects. For $\rho_{\min }<\rho<\rho_{\max }$, as the pressure is decreased, the presence of defects and interstitial water decrease, the tetrahedral structure dominates, with stronger hydrogen bonds. This process reaches its maximum at $\rho=\rho_{\text {min }} \approx \rho_{\text {ice }}$. Further stretching destabilizes the hydrogen bond network, leading to an increase in mobility. The locus

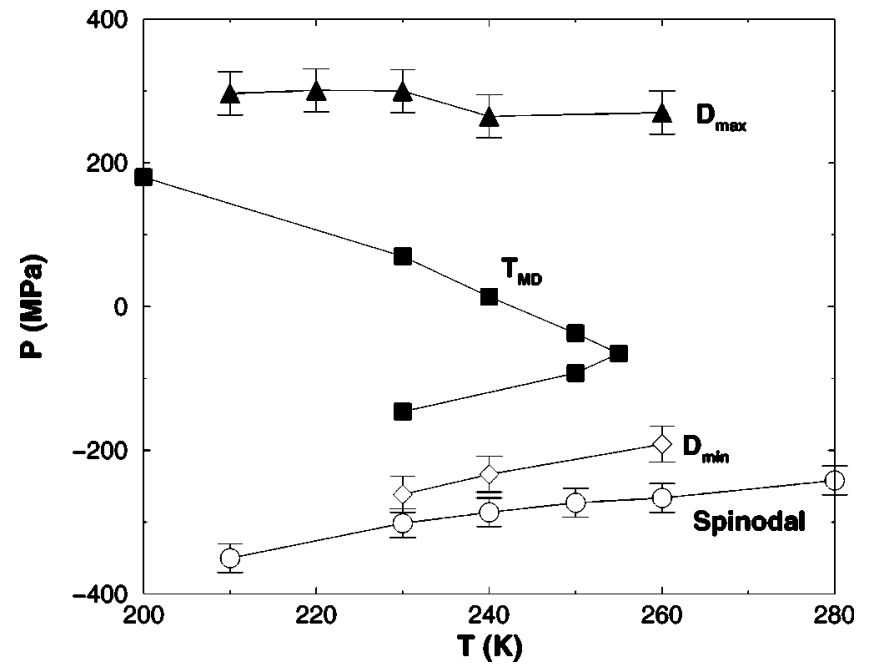

FIG. 5. Relation of the loci of maxima and minima of $D$ with $T_{M D}(P)$ and the spinodal. Open symbols are from the present work, and closed symbols are from Ref. 29. There is no maximum and minimum of $D$ for temperatures $T \geqslant 280$ K Fig. 4 and Ref. 29.

of $D_{\min }$ roughly tracks the spinodal, not surprising since the same breakdown of tetrahedral order that gives rise to $D_{\text {min }}$ also facilitates cavitation.

\section{ACKNOWLEDGMENTS}

Discussions with P. G. Debenedetti are gratefully acknowledged. The authors thank the National Science Foundation (NSF), the Conselho Nacional de Desenvolvimento Científico e Tecnológico (CNPq), the Fundação de Amparo a Pesquisa do Rio Grande do Sul (Fapergs) for financial support. F.W.S. thanks the National Research Council for financial support.

${ }^{1}$ P. G. Debenedetti, Metastable Liquids (Princeton University Press, Princeton, NJ, 1996).

${ }^{2}$ O. Mishima and H. E. Stanley, Nature (London) 396, 329 (1998).

${ }^{3}$ Supercooled Liquids: Advances and Novel Applications edited by J. T. Fourkas, D. Kivelson, U. Mohanty, and K. A. Nelson (ACS Books, Washington, DC, 1997).

${ }^{4}$ H. E. Stanley, L. Cruz, S. T. Harrington, P. H. Poole, S. Sastry, F. Sciortino, F. W. Starr, and R. Zhang, Physica A 236, 19 (1997).

${ }^{5}$ E. W. Lang and H.-D. Lüdemann, Angew. Chem. Int. Ed. Engl. 21, 315 (1982).

${ }^{6}$ R. C. Dougherty and L. N. Howard, J. Chem. Phys. 109, 7379 (1998).

${ }^{7}$ A. Geiger, F. H. Stillinger, and A. Rahman, J. Chem. Phys. 70, 4185 (1979).

${ }^{8}$ H. E. Stanley and J. Teixeira, J. Chem. Phys. 73, 3404 (1980).

${ }^{9}$ R. J. Speedy, J. Phys. Chem. 86, 982 (1982) 86, 3002 (1982).

${ }^{10}$ R. J. Speedy, J. Phys. Chem. 91, 3354 (1987).

${ }^{11}$ P. H. Poole, F. Sciortino, U. Essmann, and H. E. Stanley, Nature (London) 360, 324 (1992); Phys. Rev. E 48, 3799 (1993); F. Sciortino, P. H. Poole, U. Essmann, and H. E. Stanley, ibid. 55, 727 (1997); S. Harrington, R. Zhang, P. H. Poole, F. Sciortino, and H. E. Stanley, Phys. Rev. Lett. 78, 2409 (1997).

${ }^{12}$ O. Mishima, J. Chem. Phys. 100, 5910 (1994).

${ }^{13}$ C. J. Roberts, A. Z. Panagiotopoulos, and P. G. Debenedetti, Phys. Rev. Lett. 77, 4386 (1996); C. J. Roberts and P. G. Debenedetti, J. Chem. Phys. 105, 658 (1996).

${ }^{14}$ M.-C. Bellissent-Funnel, Europhys. Lett. 42, 161 (1998); O. Mishima and H. E. Stanley, Nature (London) 392, 192 (1998).

${ }^{15}$ H. Tanaka, J. Chem. Phys. 105, 5099 (1996).

${ }^{16}$ A. Scala, F. W. Starr, E. La Nave, H. E. Stanley, and F. Sciortino, Phys. Rev. E 62, 8016 (2000). 
${ }^{17}$ S. Sastry, P. G. Debenedetti, F. Sciortino, and H. E. Sanley, Phys. Rev. E 53, 6144 (1996).

${ }^{18}$ L. P. N. Rebelo, P. G. Debenedetti, and S. Sastry, J. Chem. Phys. 109, 626 (1998).

${ }^{19}$ P. A. Netz and Th. Dorfmüller, J. Phys. Chem. B 102, 4875 (1998).

${ }^{20}$ K. Koga, X. C. Zeng, and H. Tanaka, Chem. Phys. Lett. 285, 278 (1998).

${ }^{21}$ W. T. Pockman, J. S. Sperry, and J. W. O'Leary, Nature (London) 378, 715 (1995).

${ }^{22}$ J. Jonas, T. DeFries, and D. J. Wilbur, J. Chem. Phys. 65, 582 (1976).

${ }^{23}$ F. X. Prielmeier, E. W. Lang, R. J. Speedy, and H.-D. Lüdemann, Phys. Rev. Lett. 59, 1128 (1987); Ber. Bunsenges. Phys. Chem. 92, 1111 (1988).

${ }^{24}$ M. Rami Reddy and M. Berkovitz, J. Chem. Phys. 87, 6682 (1987).

${ }^{25}$ F. Sciortino, A. Geiger, and H. E. Stanley, Nature (London) 354, 218 (1991); J. Chem. Phys. 96, 3857 (1992).

${ }^{26}$ L. A. Baez and P. Clancy, J. Chem. Phys. 101, 9837 (1994).

${ }^{27}$ S. Harrington, P. H. Poole, F. Sciortino, and H. E. Stanley, J. Chem. Phys. 107, 7443 (1997).

${ }^{28}$ P. Gallo, F. Sciortino, P. Tartaglia, and S.-H. Chen, Phys. Rev. Lett. 76, 2730 (1996); F. Sciortino, P. Gallo, P. Tartaglia, and S.-H. Chen, Phys.

Rev. E 54, 6331 (1996); S.-H. Chen, P. Gallo, F. Sciortino, and P.
Tartaglia, ibid. 56, 4231 (1997); F. Sciortino, L. Fabbian, S.-H. Chen, and P. Tartaglia, ibid. 56, 5397 (1997).

${ }^{29}$ F. W. Starr, F. Sciortino, and H. E. Stanley, Phys. Rev. E 60, 6757 (1999); F. W. Starr, S. T. Harrington, F. Sciortino, and H. E. Stanley, Phys. Rev. Lett. 82, 3629 (1999).

${ }^{30}$ A. Scala, F. W. Starr, E. La Nave, F. Sciortino, and H. E. Stanley, Nature (London) 406, 166 (2000).

${ }^{31}$ S. J. Henderson and R. J. Speedy, J. Phys. E 13, 778 (1980).

${ }^{32}$ J. L. Green, D. L. Durben, G. H. Wolf, and C. A. Angell, Science 249, R649 (1990).

${ }^{33}$ I. I. Vaisman, L. Perera, and M. L. Berkovitz, J. Chem. Phys. 98, 9859 (1993).

${ }^{34}$ P. Maunsbach, J. Schnitker, and A. Geiger, Z. Angew. Math. Phys. 74, T608 (1994)

${ }^{35}$ J. R. Errington and P. G. Debenedetti, Nature (London) 409, 318 (2001).

${ }^{36}$ Note that while $S(q)$ clearly indicates phase separation in the system, the static pair correlation function $g(r)$ [the Fourier transform of $S(q)$ ] in the unstable region of the phase diagram shows no significant difference from $g(r)$ in the metastable region. 